\title{
Xanthine Oxidoreductase Activity in Platelet-Poor and Rich Plasma As a Oxidative Stress Indicator in Patients Required Renal Replacement Therapy
}

Elżbieta Cecerska-Heryć ( $\sim$ cecerskaela@wp.pl)

Pomorski Uniwersytet Medyczny w Szczecinie https://orcid.org/0000-0002-7313-7193

Rafał Heryć

Pomeranian Medical University in Szczecin: Pomorski Uniwersytet Medyczny w Szczecinie

Grażyna Dutkiewicz

Pomeranian Medical University in Szczecin: Pomorski Uniwersytet Medyczny w Szczecinie

Anna Michalczyk

Pomeranian Medical University in Szczecin: Pomorski Uniwersytet Medyczny w Szczecinie

Bartłomiej Grygorcewicz

Pomeranian Medical University in Szczecin: Pomorski Uniwersytet Medyczny w Szczecinie

Natalia Serwin

Pomeranian Medical University in Szczecin: Pomorski Uniwersytet Medyczny w Szczecinie

Sylwia Napiontek-Balińska

Pomeranian Medical University in Szczecin: Pomorski Uniwersytet Medyczny w Szczecinie

\section{Barbara Dołęgowska}

Pomeranian Medical University in Szczecin: Pomorski Uniwersytet Medyczny w Szczecinie

\section{Research article}

Keywords: Xanthine oxidoreductase, Platelets, Renal Replacement Therapy, Chronic Kidney Disease, Antioxidant Enzymes

Posted Date: April 6th, 2021

DOl: https://doi.org/10.21203/rs.3.rs-390059/v1

License: (c) (i) This work is licensed under a Creative Commons Attribution 4.0 International License. Read Full License

Version of Record: A version of this preprint was published at BMC Nephrology on January 18th, 2022. See the published version at https://doi.org/10.1186/s12882-021-02649-8. 


\section{Abstract}

Background: Xanthine oxidoreductase (XOR) is a hydroxylase enzyme involved in the metabolism of purines. XOR activity can vary as the homodimer protein can be converted into two different isoforms: XD (antioxidant) and $\mathrm{XO}$ (prooxidant). Oxidative stress and inflammation that accompany chronic kidney disease (CKD), dialysis, and kidney transplantation result in platelet activation. This study aimed to determine the influence of applied renal replacement therapy on the activity of xanthine oxidoreductase and its isoforms.

Methods: The study group consisted of 117 patients divided into 4 groups: hemodialysis (30), peritoneal dialysis (30), kidney transplant patients (27) and conservative treatment (30). The control group consisted of 30 healthy volunteers.

Results: Significant differences were found in XOR activity in platelet-poor plasma (PPP) within the groups studied ( $p=0.001)$. There was a relationship between type of renal replacement therapy and the oxidoreductase isoforms in PPP ( $p<0.001$ all isoforms), and XD $(p=0.008)$ and XO $(p<0.001)$ in PRP. An association was observed between the activity of all oxidoreductase isoforms in PPP and PRP, and type of renal replacement therapy, duration of dialysis and the age of patients. The presence of CKD also reflected differences in XD and XO activity in PPP.

Conclusions: The type of renal replacement therapy used in CKD patients, age of patients, duration of dialysis, CKD causes, and stage of progression significantly affect the activity of XOR and its isoforms.

\section{Background}

Xanthine oxidoreductase (XOR) is a hydroxylase enzyme involved in the metabolism of purines. It catalyzes the oxidation of hypoxanthine to xanthine, and xanthine to uric acid (UA). XOR activity can vary as the homodimer protein can be converted into two different isoforms: Xanthine dehydrogenase (XD) which is expressed predominantly in healthy tissue, and xanthine oxidase $(\mathrm{XO})$ generated by posttranslational XD modification, oxidation of cysteine residues as well as limited proteolysis, which plays a dominant role in cells and tissues during injuries [1,2,3]. These isoforms have opposing activities [4]. XOR acts in the presence of $\mathrm{NAD}^{+}$as a dehydrogenase and with molecular oxygen as an oxidase. The ability of XOR to rapidly convert from antioxidant to oxidant in various types of tissue damage is an essential element for a rapid innate immune response, beneficial in, for example, bacterial or fungal infection [5]. A reaction intermediate of xanthine dehydrogenase and oxygen (XDO) can react with both $\mathrm{NAD}^{+}$and $\mathrm{O}_{2}$, but demonstrates a higher affinity for $\mathrm{NAD}^{+}[6]$. This intermediate isoform has not been isolated, but determination of its activity facilitates the tracking of the transformation of XD to XO [7].

Serum XOR activity in various diseases has been widely investigated. The reason for interest in this enzyme is the dualism of its action: the ability to produce antioxidants, and on the other hand, generation of reactive oxygen species (ROS). An increase in XOR activity occurs in pathological conditions such as 
viral hepatitis, infectious mononucleosis, autoimmune diseases, pneumonia, schizophrenia, and type II diabetes. An increase in XOR activity is also observed in the serum of patients after renal or liver transplantation [8]. It has been shown that the gene coding for XOR may be responsible for renal maturation, adipogenesis in the kidneys, and may prevent the transformation of epithelial cells into mesenchymal tissue [9]. However, to the best of our knowledge, there are no published reports that have investigated XOR activity in platelet-poor plasma (PPP) and platelet-rich plasma (PRP) in patients undergoing renal replacement therapy. The importance of XOR activity in this group of patients is related to increased platelet activation, which is caused by the oxidative stress and inflammation that accompany chronic kidney disease (CKD), dialysis, and kidney transplantation. During dialysis and organ transplantation, tissues and blood vessels become damaged and platelets are the first cells to reach the site of tissue damage, actively participating in the initial stages of the inflammatory process and healing [10].

To date, a limited number of studies have characterized XOR and the activities of its isoforms in plateletrich or poor plasma in patients suffering from CKD. Tan et al. showed cells damaged by ROS (in the case of chronic renal replacement therapy) "leak" XOR isoforms, leading to an increase in levels of the enzyme in plasma [11]. This mechanism explains the lower activity of XOR and its isoforms in platelets compared to PPP. Therefore, the activity of oxidoreductases in PPP and PRP is of great interest, as it could help to uncover the cellular processes that occur during dialysis. Such investigations could also indirectly highlight the severity of oxidative stress in this group of patients.

Our research team has been studying the topic of XOR activity in PPP and PRP plasma for several years now. We are the only group that studies the activity of XO, XD, and XDO isoforms in plasma. In 2015, we conducted a study on XOR activity among healthy volunteers. The results were impressive. The healthy volunteers showed highest activity of the $\mathrm{XO}$ isoform (prooxidant) and low activity of the XD isoform (antioxidant), which indicates slight oxidative stress among the patients that were tested, confirming the physiological effects of XOR [12].

In 2017, we continued research among a group of hemodialysis patients. The study showed that hemodialysis patients are exposed to very strong oxidative stress, as evidenced by the highest activity of the XD and XO isoforms. This stress was, however, partially compensated. We showed a decrease in oxidative stress after hemodialysis, as evidenced by a reduction of XO activity and an increase in XD activity in PPP plasma. These studies encouraged us to investigate the effect of renal replacement therapy on XOR activity [13].

Based on the activity of antioxidant enzymes, we can determine which type of renal replacement therapy is less likely to expose the patient to oxidative stress. Due to the dual nature of XOR, the understanding of the relationship between type of renal replacement therapies and the activity of XOR isoforms can be very interesting and helpful in therapy selection. We present the results of this study in this work.

\section{Methods}




\section{Ethics approval and consent}

The Bioethical Commission at the Pomeranian Medical University in Szczecin approved the research study (no. KB-0012/36/11). All participants, including the healthy volunteers in the control group, were informed about the purpose and scope of the study and gave their consent to donation of samples and publication of the resulting data.

\section{Study group}

There were 117 participants with chronic kidney disease (CKD) in the study group. The patients were divided into 4 groups based on the treatment they received: 30 patients before and after hemodialysis (HD A and $\mathrm{HD} \mathrm{B}$ ): 30 received peritoneal dialysis (PD); 27 before and after kidney transplantations (5-7 days after surgery) (TE A, TE B); and 30 received conservative treatment (CKD) (CKD stage 2-5). The control group consisted of 30 healthy volunteers. All study participants gave their written consent to participate in the study. Additional information characterizing the study group are presented in Tables 1 and 2. $[14,15]$. 
Table 1

General characteristics of hemodialysis patients (HD), peritoneal dialysis (PD) treated conservatively (CKD), kidney transplantation (TE) and control group (C) participating in the study (mean \pm OS)

\begin{tabular}{|c|c|c|c|c|c|c|c|}
\hline Parameters & HD & PD & CKD & TE & NK & $\mathrm{p}^{*}$ & $p^{\star \star}$ \\
\hline Gender & M-18 & M-16 & M-17 & $M-14$ & M-18 & NS & NS \\
\hline [M-male; & F-12 & F-14 & $\mathrm{F}-13$ & F-13 & $\mathrm{F}-12$ & & \\
\hline \multicolumn{8}{|l|}{ F - female] } \\
\hline Age [years] & $63 \pm 16$ & $55 \pm 15$ & $66 \pm 15$ & $57 \pm 11$ & $\begin{array}{l}50 \pm \\
8\end{array}$ & $<0,001$ & 0,029 \\
\hline $\begin{array}{l}\text { Dialysis duration } \\
\text { [months] }\end{array}$ & $25 \pm 16$ & $26 \pm 22$ & - & $54 \pm 34$ & - & - & 0,003 \\
\hline Causes of CKD & $5(17 \%)$ & $5(17 \%)$ & $\begin{array}{l}4 \\
(13 \%)\end{array}$ & $1(4 \%)$ & - & - & NS \\
\hline $2-\mathrm{HA}$ & $\begin{array}{l}15 \\
(50 \%)\end{array}$ & $3(10 \%)$ & $\begin{array}{l}6 \\
(20 \%)\end{array}$ & $0(0 \%)$ & - & - & NS \\
\hline $3-\mathrm{KZN}$ & $2(7 \%)$ & $9(30 \%)$ & $\begin{array}{l}6 \\
(20 \%)\end{array}$ & $3(11 \%)$ & - & - & NS \\
\hline $4-\mathrm{ADPKD}$ & $0(0 \%)$ & $0(0 \%)$ & $\begin{array}{l}4 \\
(13 \%)\end{array}$ & $2(7 \%)$ & - & - & NS \\
\hline 5 - others & $5(17 \%)$ & $\begin{array}{l}10 \\
(33 \%)\end{array}$ & $\begin{array}{l}4 \\
(13 \%)\end{array}$ & $6(22 \%)$ & - & - & NS \\
\hline 6 - unknown & $3(10 \%)$ & $3(10 \%)$ & $\begin{array}{l}6 \\
(20 \%)\end{array}$ & $\begin{array}{l}15 \\
(56 \%)\end{array}$ & - & - & NS \\
\hline \multicolumn{8}{|c|}{$\begin{array}{l}P^{*} \text { - statistical significance for differences between HD, PD and CT groups, TE and NK exact Fisher } \\
\text { test for qualitative variables; for quantitative variables - one-way ANOVA and; }\end{array}$} \\
\hline \multicolumn{8}{|c|}{$\begin{array}{l}\text { P ** - statistical significance for differences between HD, PD and CT groups and TE exact Fisher test } \\
\text { for qualitative variables for quantitative variables - one-way ANOVA or; DM - diabetic nephropathy; HA } \\
\text { - hypertension; GIK - glomerular inflammation kidney; ADPKD - polycystic kidney disease inherited } \\
\text { autosomal dominant; NS - no statistically significant differences. }\end{array}$} \\
\hline
\end{tabular}


Table 2

General characteristics of hemodialysis patients ( $B$ - before HD, A - after), peritoneal dialysis (PD) treated conservatively (CKD)before and after kidney transplantation (TE B and TE A) and control group (NK) taking part in the study (mean \pm OS).

\begin{tabular}{|lllllllllll|}
\hline Parameters & HD B & HD & PD & CKD & TE B & TE A & C & P* & P** \\
\hline Kt/V & $1,3 \pm$ & - & $2,8 \pm$ & - & - & - & - & - & < \\
& 0,2 & & 1,06 & & & & & & 0,001 \\
Concentration & $7,9 \pm$ & 3,5 & $4,4 \pm$ & $2,5 \pm$ & $7,4 \pm$ & 3,4 & $0,8 \pm$ & $<$ & $<$ \\
of creatinine \\
[mg/dl]
\end{tabular}

$P$ * - statistical significance for differences between HD, PD and CT groups, TE and NK for quantitative variables - Kruskal Wallis ANOVA, ANOVA one-way ANOVA or Student's t test

$P$ ** - statistical significance for differences between HD, PD and CT and TE groups for Kruskal Wallis's ANOVA quantitative variables or ANOVA one-way analysis.

$\mathrm{Kt} / \mathrm{V}$ - dialysis index (volume fraction $\mathrm{V}$ purified by clearance $\mathrm{K}$ at time $\mathrm{t}$ )

NS - no statistically significant relationships were found 
Blood samples ( $\mathrm{K}_{2}$ EDTA $8 \mathrm{~mL}, 3.8 \%$ trisodium citrate 9: 1; $\mathrm{v} / \mathrm{v}$ and serum $8 \mathrm{~mL}$ ) were drawn from all study participants. Hemodialyzed patient blood was drawn from the arteriovenous fistula; peripheral venipuncture was used for all other participants. Samples were taken from hemodialysis patients before (HD A) and about 10 minutes after the pump was stopped (HD B). Transplant patient blood was collected before transplantation (TE A) and 5-7 days after surgery (TE B). Patients recruited for the TE group did not belong to the group of hemodialysis or peritoneal dialysis patients in this study - they were patients qualified for transplantation from all over Poland. The vast majority of patients with kidney transplantation had prior hemodialysis. $\mathrm{K}_{2}$ EDTA and clotted blood samples were centrifuged at 2,600 rpm for 10 minutes at $20^{\circ} \mathrm{C}$ to obtain plasma and serum, respectively. In order to obtain platelet-rich plasma (PRP) and platelet poor-plasma (PPP), blood collected with citrate was centrifuged at 1,100 rpm for 10 minutes at $20^{\circ} \mathrm{C}$. The resulting plasma was transferred to a new tube and centrifuged at $5,000 \mathrm{rpm}$ for 10 minutes at $20^{\circ} \mathrm{C}$, followed by transfer of platelet-poor plasma (PPP) to a separate tube after which the platelet pellet was rinsed twice and suspended in Tyrode's buffer ( $\mathrm{pH}$ 7.4). Plasma, serum, PPP, and PRP were frozen at $-80^{\circ} \mathrm{C}$ until the assays were performed $[12,13,14,15]$.

\section{Xanthine oxidoreductase activity in platelet-poor plasma and platelets}

Enzyme activities were measured using a Perkin Elmer UV/VIS Lambda 40P spectrophotometer. Extinction changes were recorded at $340 \mathrm{~nm}(X D)$ and $302 \mathrm{~nm}(X D O, X O)$ for 5 minutes at $30^{\circ} \mathrm{C}$. The enzymatic activity was measured as the formation of uric acid and $\mathrm{NADH}$ (increases in $\mathrm{A}_{340}$ and $\mathrm{A}_{302}$ )

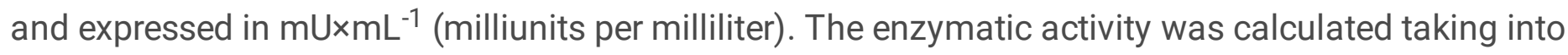
account the initial rates of reaction. Uric acid formation was measured at $302 \mathrm{~nm}$ (isoforms XDO and XO) because its absorbance is still high at this wavelength, whereas changes in $\mathrm{NAD}^{+}$concentration do not contribute. The extinction coefficient for $\mathrm{NADH}^{+} \mathrm{H}^{+} \varepsilon 340=6.22 \times 10^{3}\left[\mathrm{~L} \cdot \mathrm{mol}^{-1} \mathrm{~cm}^{-1}\right]$ was used to calculate

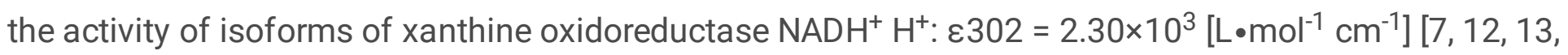
$14,15,17]$.

\section{Statistical analyses}

To assess distributions, the K-S test was used, which in the case of some variables, (the activity of XD and XDO isoforms in PRP) showed a non-normal distribution of parameters. Exact Fisher and Chi-square tests were used to analyze quantitative data. Using the Student's t-test and ANOVA analysis for univariate systems, the differences between associated (paired) and unrelated (unpaired) variables were evaluated for variables with a normal distribution. For variables with non-normal distributions, Kruskal-Wallis ANOVA analysis was performed to evaluate differences between the parameters, as well as the MannWhitney U nonparametric test for unpaired data or Wilcoxon for paired data. A linear multiple regression model was used to determine the multifactor evaluation of relationships between the parameters studied. Statistical analysis of results was performed using Statistica 12 (StatSoft) $[14,15]$. 


\section{Results}

\section{The activity of xanthine oxidoreductase in platelet-poor and platelet-rich plasma}

Significant differences were found in XOR activity in platelet-poor plasma and in platelets within the groups studied (Fig. 1A, B): the highest mean activity of oxidoreductase was seen in the control group (also in PPP and in PRP), lower before hemodialysis (HD A), and the lowest after kidney transplantation (TE A) (PPP) and in patients treated conservatively (PRP).

Differences in the activity of the XD isoform in PPP between studied groups were also observed (Table 3). The lowest XD activity was observed in the group of kidney transplant patients, and the highest in the control group (Fig. 2A). There was a significant correlation between the measured XDO activities in the PPP in the groups (Table 3). The lowest activity of the intermediate isoform was demonstrated in patients after renal transplantation (TE A), and the highest in the group of patients treated conservatively (CT) (Fig. 2B). The relationship between the activity of the XO isoform between the groups in PPP (Table 3) approached significance, with the lowest $\mathrm{XO}$ activity observed in the group of patients after kidney transplantation, and the highest before hemodialysis (Fig. 2C). Other relationships between particular groups are shown in Table 4.

Table. 3 Activity of XOR isoforms in the platelet-poor plasma of patients with chronic renal hemodialysis patients (before and after $H D B, H D A$ ), peritoneal dialysis (PD), conservative treatment (CKD), before and after kidney transplantation (TE B, TE A) and in the control group (C) (mean \pm OS, median - lower and upper quartile). 


\begin{tabular}{|llll|}
\hline $\begin{array}{l}\text { Activity of XOR isoforms } \\
\text { [mU/mL] } \\
\text { Groups }\end{array}$ & XD XDO & \multicolumn{1}{c|}{ XO } & \\
\hline HD B & & & \\
& $42,5 \pm 14,5$ & $8,7 \pm 5,8$ & $67,2 \pm 24,5$ \\
\hline HD A & $42,7(10,0 ; 68,0)$ & $8,6(0,6 ; 26,2)$ & $68,0(22,6 ; 122,8)$ \\
\hline DO & $45,6 \pm 16,8$ & $7,6 \pm 4,6$ & $58,5 \pm 23,4$ \\
& $44,5(9,6 ; 83,6)$ & $7,8(0,08 ; 15,9)$ & $57,3(16,5 ; 94,6)$ \\
\hline CKD & $34,6 \pm 26,1$ & $8,7 \pm 6,9$ & $46,9 \pm 20,5$ \\
& $28,1(5,2 ; 116,5)$ & $6,8(0,1 ; 27,7)$ & $43,3(9,4 ; 85,0)$ \\
\hline TE B & $35,8 \pm 20,4$ & $19,4 \pm 17,5$ & $53,2 \pm 72,6$ \\
& $37,3(4,8 ; 88,5)$ & $17,6(0,9 ; 75,3)$ & $40,4(1,3 ; 423,2)$ \\
\hline TE A & $33,8 \pm 16,7$ & $11,3 \pm 6,2$ & $40,3 \pm 25,2$ \\
& $33,3(7,4 ; 75,1)$ & $10,5(1,6 ; 26,0)$ & $33,9(3,9 ; 124,4)$ \\
\hline C & $22,1 \pm 13,8$ & $7,1 \pm 3,4$ & $39,1 \pm 49,3$ \\
& $17,5(2,2 ; 53,6)$ & $6,7(1,3 ; 14,7)$ & $28,4(5,8 ; 274,0)$ \\
\hline P & $47,0 \pm 15,5$ & $14,8 \pm 6,9$ & $58,5 \pm 15,4$ \\
\hline
\end{tabular}

Table 4. Statistical differences in the activity of XOR isoforms in PPPs between the studied groups ( $p$ value) 


\begin{tabular}{|c|c|}
\hline \multicolumn{2}{|l|}{$X D$ in $P P P$} \\
\hline Gropus & $P$ value \\
\hline$H D B$ vs TE $B$ & 0,04 \\
\hline$H D B$ vs TE $A$ & $<0,001$ \\
\hline$H D A$ vs $C T$ & 0,044 \\
\hline$H D A$ vs TE B & 0,01 \\
\hline$H D A$ vs TE $B$ & $<0,001$ \\
\hline$P D$ vs TE $B$ & 0,031 \\
\hline$P D$ vs $C$ & 0,031 \\
\hline$C K D$ vs TE B & 0,005 \\
\hline$T E A$ vs TE $B$ & 0,004 \\
\hline$T E B$ vs $C$ & 0,005 \\
\hline$T E A$ vs $C$ & $<0,001$ \\
\hline \multicolumn{2}{|l|}{$X D O$ in $P P P$} \\
\hline Gropus & $P$ value \\
\hline$H D B$ vs $C K D$ & 0,002 \\
\hline$H D B$ vs $C$ & $<0,001$ \\
\hline$H D A$ vs $C K D$ & $<0,001$ \\
\hline$H D A$ vs TE $B$ & 0,014 \\
\hline$H D A v s C$ & $<0,001$ \\
\hline$P D$ vs $C K D$ & 0,02 \\
\hline$P D$ vs $C$ & 0,001 \\
\hline$C K D$ vs TE B & 0,025 \\
\hline CKD vs TE $A$ & $<0,001$ \\
\hline$T E B$ vs TE $A$ & 0,011 \\
\hline$T E B$ vs $C$ & 0,05 \\
\hline$T E B$ vs $C$ & $<0,001$ \\
\hline
\end{tabular}

Page 10/25 


\begin{tabular}{|ll|}
\hline XO in PPP & \\
\hline$H D B$ vs $P D$ & $<0,001$ \\
\hline$H D B$ vs TE $B$ & $<0,001$ \\
\hline$H D B$ vs TE $A$ & 0,008 \\
\hline$H D A$ vs $P D$ & 0,042 \\
\hline$H D A$ vs TE $B$ & 0,006 \\
\hline$P D$ vs $C$ & 0,016 \\
\hline$T E B$ vs $C$ & 0,002 \\
\hline$T E A$ vs $C$ & 0,048 \\
\hline
\end{tabular}

A significant difference was found between the activities of XD, XDO, and $X O$ isoforms in PPP among the examined groups (Figure $2 A, B, C$ ). There was a significant difference in the activity of the XD dehydrogenase isoform in the PRP between groups (Table 5): the lowest XD activity was found in patients treated conservatively, and the highest in patients prior to hemodialysis (Figure 3A). There was a significant difference in the activity of the XO isoform in PRP between the study groups (Table 5); the lowest XO activity was found in patients treated conservatively, and the highest in patients before renal transplantation (Figure 3C). Other relationships between the activity of all XOR isoforms and type of renal replacement therapy are included in Table 6 . Additionally, a difference was found between the activities of XD, XDO, and XO isoforms in PRP between the test groups (Table 6)

Table. 5 Activity of XOR isoforms in the platelet-rich plasma of patients with chronic renal hemodialysis patients (before and after HD B, HD A), peritoneal dialysis (PD), conservative treatment (CKD), before and after kidney transplantation (TE B, TE A) and in the control group (C) (mean \pm OS, median - lower and upper quartile). 


\begin{tabular}{|llll|}
\hline $\begin{array}{l}\text { Activity of XOR isoforms } \\
\text { [mU/mL] } \\
\text { Groups }\end{array}$ & XD XDO & \multicolumn{1}{c|}{ XO } & \\
\hline HD B & & & \\
& $19,7 \pm 14,3$ & $4,8 \pm 5,6$ & $18,6 \pm 9,3$ \\
\hline HD A & $16,8(1,6 ; 54,1)$ & $2,9(0,3 ; 28,4)$ & $18,3(1,9 ; 38,8)$ \\
\hline PD & $15,9 \pm 14,4$ & $6,0 \pm 4,5$ & $15,9 \pm 8,7$ \\
& $8,6(0,8 ; 51,7)$ & $4,8(0,1 ; 15,6)$ & $14,1(3,9 ; 38,1)$ \\
\hline CKD & $9,6 \pm 6,7$ & $7,1 \pm 4,3$ & $24,0 \pm 9,5$ \\
& $8,2(0,8 ; 32,8)$ & $6,9(0,08 ; 15,1)$ & $23,9(6,8 ; 43,9)$ \\
\hline TE B & $18,0 \pm 18,2$ & $4,0 \pm 3,1$ & $10,4 \pm 8,3$ \\
& $7,4(1,6 ; 69,7)$ & $3,3(0,1 ; 11,8)$ & $9,0(0,6 ; 38,4)$ \\
\hline TE A & $13,6 \pm 14,8$ & $6,4 \pm 6,1$ & $24,7 \pm 18,2$ \\
& $9,3(3,3 ; 79,5)$ & $4,9(0,4 ; 26,0)$ & $21,8(3,2 ; 78,2)$ \\
\hline C & $9,6 \pm 5,0$ & $6,7 \pm 4,0$ & $23,4 \pm 13,1$ \\
& $8,2(3,3 ; 18,6)$ & $6,0(1,9 ; 18,0)$ & $23,3(4,5 ; 43,9)$ \\
\hline P & $17,5 \pm 13,6$ & $5,9 \pm 4,5$ & $19,4 \pm 12,3$ \\
\hline
\end{tabular}

NS- No statistical significance

Table 6. Statistical differences in the activity of XOR isoforms in PRPs between the studied groups (pvalue) 


\begin{tabular}{|c|c|}
\hline \multicolumn{2}{|l|}{$X D$ in $P R P$} \\
\hline Gropus & $P$ value \\
\hline$H D A$ vs $H D B$ & 0,022 \\
\hline$H D B$ vs $P D$ & $<0,001$ \\
\hline$H D B$ vs TE $B$ & 0,043 \\
\hline$H D B$ vs TE $A$ & $<0,001$ \\
\hline$P D$ vs $C$ & $<0,001$ \\
\hline TE $B$ vs $C$ & 0,024 \\
\hline TE $A$ vs $C$ & 0,004 \\
\hline \multicolumn{2}{|l|}{$X D O$ in $P R P$} \\
\hline Gropus & $P$ value \\
\hline$P D$ vs $C K D$ & 0,002 \\
\hline$C K D$ vs TEA & 0,006 \\
\hline \multicolumn{2}{|l|}{$X O$ in PRP } \\
\hline Gropus & $P$ value \\
\hline$H D B$ vs $P D$ & 0,03 \\
\hline$H D B$ vs $C K D$ & $<0,001$ \\
\hline$H D A$ vs $C K D$ & 0,001 \\
\hline$H D A$ vs $P D$ & 0,014 \\
\hline HD A vs TE $B$ & 0,021 \\
\hline HD A vs TE $A$ & 0,011 \\
\hline$H D A$ vs $C$ & 0,049 \\
\hline$P D$ vs $C$ & $<0,001$ \\
\hline$C K D$ vs TE $B$ & $<0,001$ \\
\hline CKD vs TE A & $<0,001$ \\
\hline$C K D$ vs $C$ & $<0,001$ \\
\hline
\end{tabular}

The activity of xanthine oxidoreductase isoforms according to gender, duration of dialysis, patient age, cause, and severity of CKD 
There was no difference in XOR isoform activity between women and men except for the XDO isoform in platelets (Table 7) - in men, the activity of this isoform was significantly higher than in women. An association was observed between the activity of all oxidoreductase isoforms in PPP and PRP plasma and type of renal replacement therapy and duration of dialysis. There were also significant differences between the activity of all tested parameters and type of renal replacement therapy and patient age (Table 7). The cause of CKD also reflected differences in XD and XO activity in PPP. Additionally, there was also a correlation between the activity of XDO and XDO isoforms in PPP and stage of CKD (Table 7) $-X D$ activity was lowest in stage 3 disease, and highest in stage 1 disease. The activity of the XDO isoform varied differently, being lowest in stage 3 and highest in stage 4.

Table 7. The influence of particular parameters on the activity of xanthine oxidase isoforms.

\begin{tabular}{|c|c|c|c|c|c|c|}
\hline Parameters & $\begin{array}{l}X D \text { in } \\
\text { PPP }\end{array}$ & $\begin{array}{l}\text { XDO } \\
\text { in } \\
\text { PPP }\end{array}$ & $\begin{array}{l}\text { XO } \\
\text { in PPP }\end{array}$ & $\begin{array}{l}\text { XD in } \\
\text { PRP }\end{array}$ & $\begin{array}{l}\text { XDO } \\
\text { in PRP }\end{array}$ & $X O$ in PRP \\
\hline Gender & NS & NS & NS & NS & 0,03 & NS \\
\hline $\begin{array}{l}\text { Renal replacement therapy and } \\
\text { duration of dialysis }\end{array}$ & 0,002 & 0,004 & $<0,001$ & $<0,001$ & 0,012 & $<0,001$ \\
\hline Renal replacement therapy and age & $<0,001$ & $<0,001$ & $<0,001$ & $<0,001$ & $<0,001$ & $<0,001$ \\
\hline Stage of CKD & $<0,001$ & 0,018 & NS & NS & NS & 0,03 \\
\hline Causes of CKD & 0,021 & NS & NS & 0,01 & NS & NS \\
\hline
\end{tabular}

The table presents $p$ values defining statistical significance. The relationship between gender, duration of dialysis, age, stage of chronic kidney disease and the causes of chronic kidney disease and the activity of XOR isoforms was assessed using one-way ANOVA.

$\mathrm{HD}$ and duration of dialysis - the relationship between the type of therapy (hemodialysis, peritoneal dialysis, patients before kidney transplantation), duration of dialysis and the activity of XOR isoforms.

$\mathrm{HD}$ and age - dependence between the studied groups (hemodialysis, peritoneal dialysis, conservative treatment, patients before kidney transplantation and control group), patients' age and XOR isoform activity.

The stage of chronic kidney disease - the relationship between the severity of chronic kidney disease based on eGFR and the activity of XOR isoforms.

The causes of chronic kidney disease - the relationship between selected causes of chronic disease and the activity of XOR isoforms.

NS - no statistically significant relationship was found. 


\section{Discussion}

\section{General Observations}

Oxidoreductases - due to their dual activities - are intriguing and highly important enzymes that allow for the uncovering of mechanisms of oxidative stress in the human body. However, the duality of XOR also means that the interpretation of its functions is more challenging than for other antioxidant enzymes.

In the present study, the highest XOR activity was observed in the control group in both PPP and PRP, meaning that this group is best at oxidative stress compensation - the greatest activity of the antioxidant isoform (XD) observed in PPP helped confirm this. Hemodialysis patients before and shortly after kidney transplantation are highly exposed to oxidative stress as a result of underlying disease, as well as types of renal replacement therapy. This highlights the fact that peritoneal dialysis causes less exposure to the harmful effects of reactive oxygen species than other renal replacement therapies.

Dołęgowska et al. (2010) investigated XOR isoform activity in plasma in individuals after kidney transplantation, divided into three groups: early, slow, and delayed function of the transplanted organ. They found an increase in XO and XOR activity in all groups, 1 and 5 minutes after transplantation. XD activity increased in the slow and delayed function groups also after 1 and 5 minutes. The highest activity was found for the XD isoform (antioxidant) and the lowest for the XO isoform (prooxidative). This was probably due to increased oxidative stress as a result of organ transplantation, which the body tries to compensate for through increased activity of the XD isoform $[18,19]$.

Increased conversion of XD to XO after kidney transplantation was shown by Kwiatkowska et al. (2010). During the 6-month observation of patients after transplantation, the group showed that the average level of XOR in serum is constantly increasing from the first day after surgery. This suggests that XOR levels reflect the degree of damage to the transplanted organ during ischemia/reperfusion (I/R). Further increases in XOR levels can be explained by immunosuppressive therapy, which includes steroids $[20,21,22]$. Herken et al. (2007) showed that after kidney transplantation, the transformation of the XD isoform into the $\mathrm{XO}$ isoform is initiated in the ischemic period and then continues after reperfusion [21].

In this study, we found that the activity of XOR in PPP after transplantation significantly decreases, similarly to the XD and XDO isoforms. The activity of the oxidase isoform also decreases both in the poor and platelet-rich plasma, but there are no statistically significant relationships. This indicates lower oxidative stress after kidney transplantation because the conversion of the XD isoform to XO is not increased (lower XDO activity after kidney transplantation). These results may indicate small amounts of damage to the transplanted organ during the I/R period. Attention should also be paid to the significantly lower activity of XOR and its isoform in patients before and after kidney transplantation compared to other forms of renal replacement therapy, as well as compared to the control group, as it suggests a lower efficiency of the antioxidant system in this group of patients. 
The optimal type of renal replacement therapy must be selected for each patient to achieve the best treatment outcomes. Many different factors impact the success of the therapy used, one of which is oxidative stress. Therefore, it is crucial to study the effect of dialysis on XOR activity.

In our study, XOR activity decreased after hemodialysis in PPP and PRP. However, it is higher in comparison to the other groups studied (except for control groups). This indicates a higher exposure to oxidative stress in this group of patients. The activity of the XD isoform in PPP increases after hemodialysis and the XDO isoform decreases, similar to the XO isoforms. However, these were not statistically significant changes. In turn, in platelets, XD activity is significantly lower after hemodialysis while $\mathrm{XO}$ activity is lower in the HD B group, and the activity of the intermediate isoform is higher after hemodialysis (albeit with no statistical significance). This indicates a low conversion of the antioxidant to prooxidative isoform, which may mean less oxidative stress after hemodialysis.

The influence of hemodialysis on XOR activity and its isoforms in PPP and platelets were studied by Cecerska-Heryć et al. (2017). They demonstrated an effect of hemodialysis on the activity of XOR and its isoforms in PPP and in platelets. They also showed a decrease in oxidative stress after hemodialysis, as evidenced by a decrease in XO activity and an increase in XD activity in PPP plasma [13].

On the other hand, Miric et al. (2013) showed higher XOR activity before hemodialysis compared to control, and increased XOR activity during renal replacement therapy in patients with GNRI (Geriatric Nutritional Risk Index) $\leq 90$ (high risk of complications and mortality due to malnutrition), and a reduction in XOR activity in patients with GNRI >90 (low risk of death due to malnutrition) for which hemodialysis is performed. This may mean that oxidoreductase is involved in hemodialysis-induced oxidative damage, which may contribute to accelerated protein destruction in patients with GNRI $\leq 90$ [23].

Boban et al. (2014) showed that total XOR activity was higher in a group of patients suffering from essential hypertension compared to patients on dialysis. The highest activity in this group was also demonstrated by the XD isoform compared to healthy control or dialysis patients. On the other hand, the activity of $\mathrm{XO}$, which mainly contributes to the production of ROS, was the highest in dialysis patients [24].

In the case of peritoneal dialysis, the results we came upon are ambiguous. In PPP, XD activity is significantly lower compared to hemodialyzed patients, as is XO activity. This could indicate a much lower exposure to oxidative stress in patients on peritoneal dialysis than those subjected to hemodialysis. However, the pattern is different in platelets. XD activity is significantly lower in patients from the group than in hemodialysis, but XO's acuteness is significantly higher in peritoneal dialysis patients.

XDO intermediate activity was higher in patients undergoing peritoneal dialysis, which may indicate an increased conversion to the antioxidant isoform. However, it should be remembered that under the influence of the renal function test (RFT), there is a leakage of oxidoreductase from cells to plasma. Therefore, the activity of XOR isoforms in platelets related to hemodialysis and peritoneal dialysis may 
disrupt this picture. During hemodialysis, there is increased activation of platelets accompanied by strong oxidative stress, resulting in ROS that can cause XOR to be released into the plasma, which may not happen during peritoneal dialysis: hence, there is much higher XO and XDO activity in patients in the PD group. Patients treated conservatively are also exposed to oxidative stress caused by underlying disease, which is demonstrated by the highest activity of the XDO isoform and the high activity of the XO isoform in PPP.

\section{Activity of xanthine oxidoreductase isoforms according to gender, duration of dialysis, patient age, cause, and stage of CKD}

Our study revealed no relationship between XOR activity and sex, except for a significant association between XDO activity in platelets. In men, XDO activity was significantly lower than in women, supporting the theory that the activity of antioxidant enzymes may be higher in women. However, the primary purpose of measuring XDO activity is to analyze XD to XO transformations. This confirms the results reported in previous studies, where such a relationship was not observed [14,21]. Previously, only Decker et al. reported higher XOR activity in male rats compared to females in a 1982 study [25].

An age effect was observed in the activity of all isoforms of XOR in PPP and platelets in all groups. Patients in the peritoneal dialysis and TE groups were, on average, younger, and XD and XO activities in PPP were lower in these patients than in the other groups. Only XO isoform activity in platelets was higher in these (younger) groups. These results are different from those obtained for other antioxidant enzymes. This might mean that this group of patients has not yet lost XOR activity under the influence of RFT. However, it is more likely that the type of implemented renal replacement therapy affects XOR activity more than age. This seems to be confirmed by the results of our multivariate regression, as well as studies by Cecerska-Heryć et al. (2017).

The relationship between the duration and type of dialysis, and XOR activity has not yet been described in the literature, particularly in PPP and PRP. However, the issue was raised in the case of other antioxidant enzymes. The reduction of antioxidant enzyme activity in hemodialyzed patients might indicate increased oxidative stress, as observed by Olszewska et al. (2004) in a group of patients receiving the same type of renal replacement therapy [26]. Pawlak et al. (2007) observed a decrease in superoxide dismutase (SOD) activity and a correlation between the duration of dialysis and SOD activity [27]. In another study performed among hemodialyzed and peritoneally dialyzed children, it was confirmed that dialysis causes an increase in oxidative stress, increasing by the next hemodialysis. Moreover, the longer the dialysis treatment took, the higher the oxidative stress that was observed [28].

In the present study, a significant relationship was found between duration of dialysis and type of renal replacement therapy used, and the activity of all XOR isoforms in both PPP and platelets. However, in PPP from longer-lasting dialysis, a decrease in the activities of the XD and XO isoforms were observed. 
Additionally, the activity of XD, XDO, and XO isoforms increases in the platelets. The XOR activity increased in platelets due to continuous activation as a result of long-term dialysis and progressive renal disease that causes aggravation of oxidative stress and ROS production, which in turn destroys blood platelets. However, the lowering of the activity of the XOR isoforms in PPP confirm the results of other researchers on the decrease in antioxidant enzymes' activities with long-term dialysis.

Our study demonstrated the effect of CKD on the activity of XD and XO in PPP. The highest activity of the XOR isoforms occurred in patients with hypertension and diabetic nephropathy, and the lowest in patients with ADPKD (autosomal dominant polycystic kidney disease). Multivariate regression analysis indicates an independent negative correlation between XO activity in PRP and the severity of chronic kidney diseases.

Since XOR plays an important role in the production of uric acid, the relationship between XOR activity and hypertension or cardiovascular risk is widely described. Elevated levels of uric acid in the serum are associated with oxidative damage in the vessel wall, inflammatory and proliferative changes of the vessels, hypertension, and impaired renal function. Raised uric acid levels might be a factor in the increased risk of cardiovascular events. Therefore, attempts are focused to block XOR activity to reduce the concentration of uric acid. The positive effect of inhibition of XOR production on the cardiovascular system has been documented [29].

Nakatani et al. (2017 showed a positive correlation between glucose, uric acid concentration, and XOR activity in patients undergoing hemodialysis. In the same study, multivariate regression analysis showed positive, independent correlations between glucose concentration and type 2 diabetes diagnosed with XOR activity. In contrast, uric acid concentration correlated positively with XOR activity in hemodialyzed patients with undiagnosed type II diabetes. This study indicates that glycemic control might decrease oxidoreductase-mediated ROS production in hemodialysis patients [30].

The high activity of XO and XD in PPP observed in this study in patients with diabetic nephropathy and hypertension confirms the increased activity of XOR in these types of diseases due to perturbed levels of glucose and uric acid, which may exacerbate CKD. For hypertensive patients, high XO and XD activities may be an indicator of an increased risk of cardiovascular events. There is no relationship between the activity of XOR and ADPKD in the literature.

\section{Conclusion}

1. The type of renal replacement therapy used in CKD patients, age of patients, duration of dialysis, CKD causes, and stage of progression significantly affect the activity of XOR and its isoforms.

2. Peritoneal dialysis patients are exposed to less oxidative stress than hemodialysis patients.

\section{Limitations}


Patients in the study group (mainly hemodialysis) took furosemide, which may increase the level of uric acid, a strong antioxidant. It is also the last product in the hypoxanthine degradation pathway, catalyzed by XOR. However, our study showed no correlation between uric acid levels and XOR activity, except for the negative correlation of the XO isoform in PPP. This correlation only confirms the physiological properties of the oxidoreductase - the higher the uric acid concentration, the lower the XO oxidative isoform activity.

\section{Kidney transplantation}

Initially, we planned to collect biological material from patients 5-7 days after kidney transplantation, followed by one month, three months, and six months after transplantation. Unfortunately, due to logistical problems, it was not possible to provide good quality material to our unit at all time points. Therefore, we decided to collect material at only one time point, which was about 7 days after transplantation.

\section{Declarations}

\section{Ethics approval and consent to participate:}

The Bioethical Commission at the Pomeranian Medical University in Szczecin approved the research carried out (no. KB-0012/36/11). All participants, including the healthy volunteers in the control group, were informed about the purpose and scope of the study and gave their consent to donate samples and for data to be published

All procedures performed in the studies involving human participants were in accordance with the ethical standards of the institutional and/or national research committee and with the 1964 Helsinki declaration and its later amendments, or comparable ethical standards.

\section{Consent for publication}

Written informed consent was obtained from patients for publication of this study.

\section{Availability of data and materials}

The datasets generated and/or analyzed during the current study are not publicly available as these are sensitive data owned by the Pomeranian Medical University in Szczecin, but are available from the corresponding author upon reasonable request.

\section{Competing interest}

The authors declare that they have no competing interests.

\section{Funding}


The paper was funded by the Pomeranian Medical University in Szczecin grant no. MB-134-141/15. The funding body covered the costs of material collection and the purchase of reagents and data analysis.

\section{Authors' contributions:}

ECH -Writing, Original Draft Preparation, Methodology

RH - Writing, Review \& Editing

GD - Investigation Resources

BG - Visualization

AM - Investigation Resources, Methodology

NS - Formal Analysis

SNB - Resources

BD - Conceptualization

All authors have read and approved the manuscript.

\section{Acknowledgments:}

Not applicable

\section{References}

1. Okamoto K., Matsumoto K., Hille R., Eger B.T., Pai E.F., Nishino T.: The crystal structure of xanthine oxidoreductase during catalysis: implications for reaction mechanism and enzyme inhibition. Proc Natl Acad Sci U S A. 2004;101:7931-6.

2. Meneshian A, Bulkley G.B.: The physiology of endothelial xanthine oxidase: from urate catabolism to reperfusion injury to inflammatory signal transduction. Microcirculation. 2002;9:161-75.

3. Dolegowska B., Blogowski W., Domanski L.: Clinical evidence of the association between serum perioperative changes in xanthine metabolizing enzymes activity and early post-transplant kidney allograft function. J Am Coll Surg. 2010;211:587-95.

4. Hille R., Nishino T.: Flavoprotein structure and mechanism. 4. Xanthine oxidase and xanthine dehydrogenase. Faseb j. 1995;9:995-1003.

5. Vorbach C., Harrison R., Capecchi M.R.: Xanthine oxidoreductase is central to the evolution and function of the innate immune system. Trends Immunol. 2003;24(9):512-7.

6. Kaminski Z.W., Jezewska M.M.: Intermediate dehydrogenase-oxidase form of xanthine oxidoreductase in rat liver. Biochem J. 1979;181:177-82. 
7. Dołęgowska B.: Predictors of delayed transplanted kidney function - analysis of prooxidation and antioxidative balance and arachidonic acid metabolism in blood platelets and plasma during ischemia-reperfusion. Szczecin: Wydawncitwo Pomorskiej Akademii Medycznej; 2009.

8. Battelli M.G, Bolognesi A., Polito L.: Pathophysiology of circulating xanthine oxidoreductase: new emerging roles for a multi-tasking enzyme. Biochim Biophys. Acta. 2014; 1842:1502-17.

9. Ohtsubo T., Matsumura K., Sakagami K., Fujii K., Tsuruya K., et al.: Xanthine oxidoreductase depletion induces renal interstitial fibrosis through aberrant lipid and purine accumulation in renal tubules. Hypertension. 2009;54:868-76.

10. Szczeklik A.(red), Diseases of the kidneys of the urinary tract. Acute kidney injuryand chronic kidney disease.,Interna Szczeklika, Medycyna Praktyczna, Kraków 2017, 1443-1461.

11. Tan S., Gelman S., Wheat J.K, Parks D.A.: Circulating xanthine oxidase in human ischemia reperfusion. South Med J. 1995; 88:479-82.

12. Cecerska-Heryc E., Jesionowska A., Klaudyna S., Katarzyna S., Dominika M., et al.: Xanthine oxidoreductase reference values in platelet-poor plasma and platelets in healthy volunteers. Oxid Med Cell Longev. 2015;2015:341926.

13. Cecerska-Heryć E., Jesionowska A.,Marczuk N., Heryć R.,Dołęgowska B.: Xanthine oxidoreductase activity, in platelet-poor plasma and in platelets, in hemodialyzed patients. Nauki medyczne i nauki o zdrowiu.2017;4:7-13.

14. Cecerska-Heryć, E., Heryć, R., Wiśniewska, M. et al. Regenerative potential of platelets in patients with chronic kidney disease. Int Urol Nephrol. 2019; 51, 1831-1840.

15. Cecerska-Heryć, E., Heryć, R., Wiśniewska, M. et al. Effect of renal replacement therapy on selected arachidonic acid derivatives concentration. BMC Nephrol. 2020; 21,

16. Stępniewska J., Dołęgowska B., Cecerska-Heryć E., Gołembiewska E., Malinowska-Jędraszczyk A., et al.: The activity of antioxidant enzymes in blood platelets in different types of renal replacement therapy: a cross-sectional study. Int Urol Nephrol. 2016 ;48:593-9.

17. Rodrigo E., Fernandez-Fresnedo G., Ruiz J.C., Pinera C., Palomar R.,et al.: Similar impact of slow and delayed graft function on renal allograft outcome and function. Transplant Proc. 2005;37:1431-2.

18. Pandey N.R., Kaur G., Chandra M., Sanwal G.G., Misra M.K.: Enzymatic oxidant and antioxidants of human blood platelets in unstable angina and myocardial infarction. Int $\mathrm{J}$ Cardiol. 2000;76:33-8.

19. Dolegowska B., Blogowski W., Domanski L.: Clinical evidence of the association between serum perioperative changes in xanthine metabolizing enzymes activity and early post-transplant kidney allograft function. J Am Coll Surg. 2010;211:587-95.

20. Reinhold S.W., Scherl T., Stölcker B., Bergler T., Hoffmann U., et al.: Lipoxygenase products in the urine correlate with renal function and body temperature but not with acute transplant rejection. Lipids. 2013;48:167-75.

21. Kwiatkowska E., Kędzierska K., Bober J., Dołęgowska B., Dziedziejko V., et al.: Urinary hepatocyte growth factor indicates ischemia/reperfusion injury after kidney transplantation. Pol Arch Med 
Wewn. 2010; 120:437-42.

22. Herken H., Gurel A., Selek S., Armutcu F., Ozen M.E., et al.: Adenosine deaminase, nitric oxide, superoxide dismutase, and xanthine oxidase in patients with major depression: impact of antidepressant treatment. Arch Med Res. 2007; 38:247-52.

23. Miric D., Kisic B., Stolic R., Miric B., Mitic R., el.: The role of xanthine oxidase in hemodialysis-induced oxidative injury: relationship with nutritional status. Oxid Med Cell Longev. 2013:245253.

24. Boban M., Kocic G., Radenkovic S., Pavlovic R., Cvetkovic T., et al.: Circulating purine compounds, uric acid, and xanthine oxidase/dehydrogenase relationship in essential hypertension and end stage renal disease. Ren Fail. 2014;36:613-8.

25. Decker D.E, Levinson D.J.: Quantitation of rat liver xanthine oxidase by radioimmunoassay. A mechanism for sex-specific differences. Arthritis Rheum. 1982;25:326-32.

26. Olszewska M.: The effect of hemodialysis on some parameters of the antioxidant system in the blood of patients with chronic renal failure. Ann. Acad. Med. Stetin. 2004; 50:41-52.

27. Pawlak K., Pawlak D., Mysliwiec M.: Impaired renal function and duration of dialysis therapy are associated with oxidative stress and proatherogenic cytokine levels in patients with end-stage renal disease. Clin. Biochem. 2007; 40:81-85.

28. Zwołińska D., Grzeszczak W., Szczepańska M., Kiliś-Pstrusińska K., Szprynger K.: Lipid peroxidation and antioxidant enzymes in children on maintenance dialysis. Pediatr Nephrol. 2006; 21:705-710.

29. Schuchardt M., Herrmann J., Tolle M., van der Giet M.:Curr Pharm Des. Xanthine Oxidase and its Role as Target in Cardiovascular Disease: Cardiovascular Protection by Enzyme Inhibition? Curr Pharm Des. 2017;23:3391-3404.

30. Nakatani A., Nakatani S., Ishimura E., Murase T., Nakamura T., et al.: Xanthine oxidoreductase activity is associated with serum uric acid and glycemic control in hemodialysis patients. Sci Rep. 2017;7:15416.

\section{Figures}


A

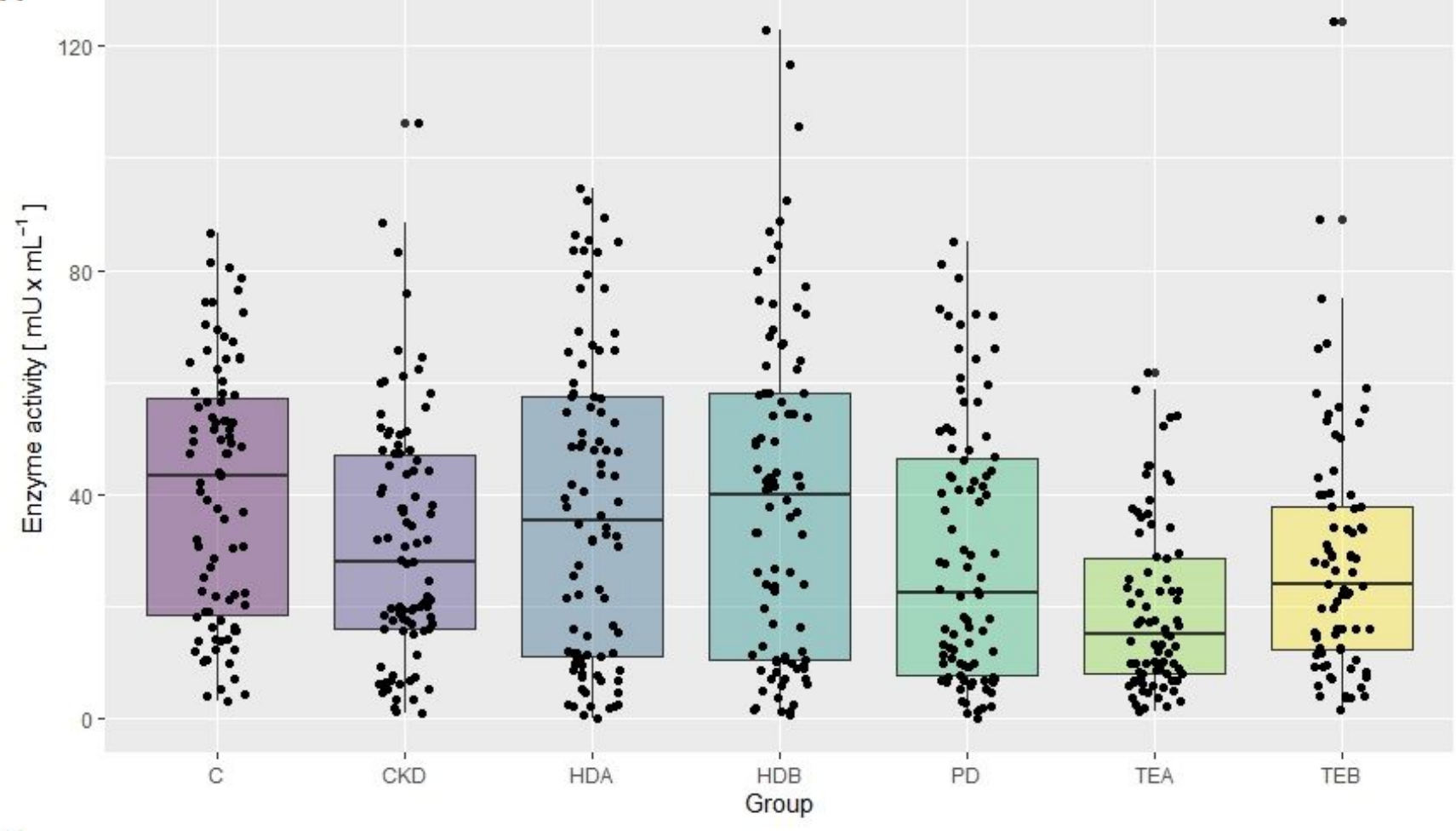

B

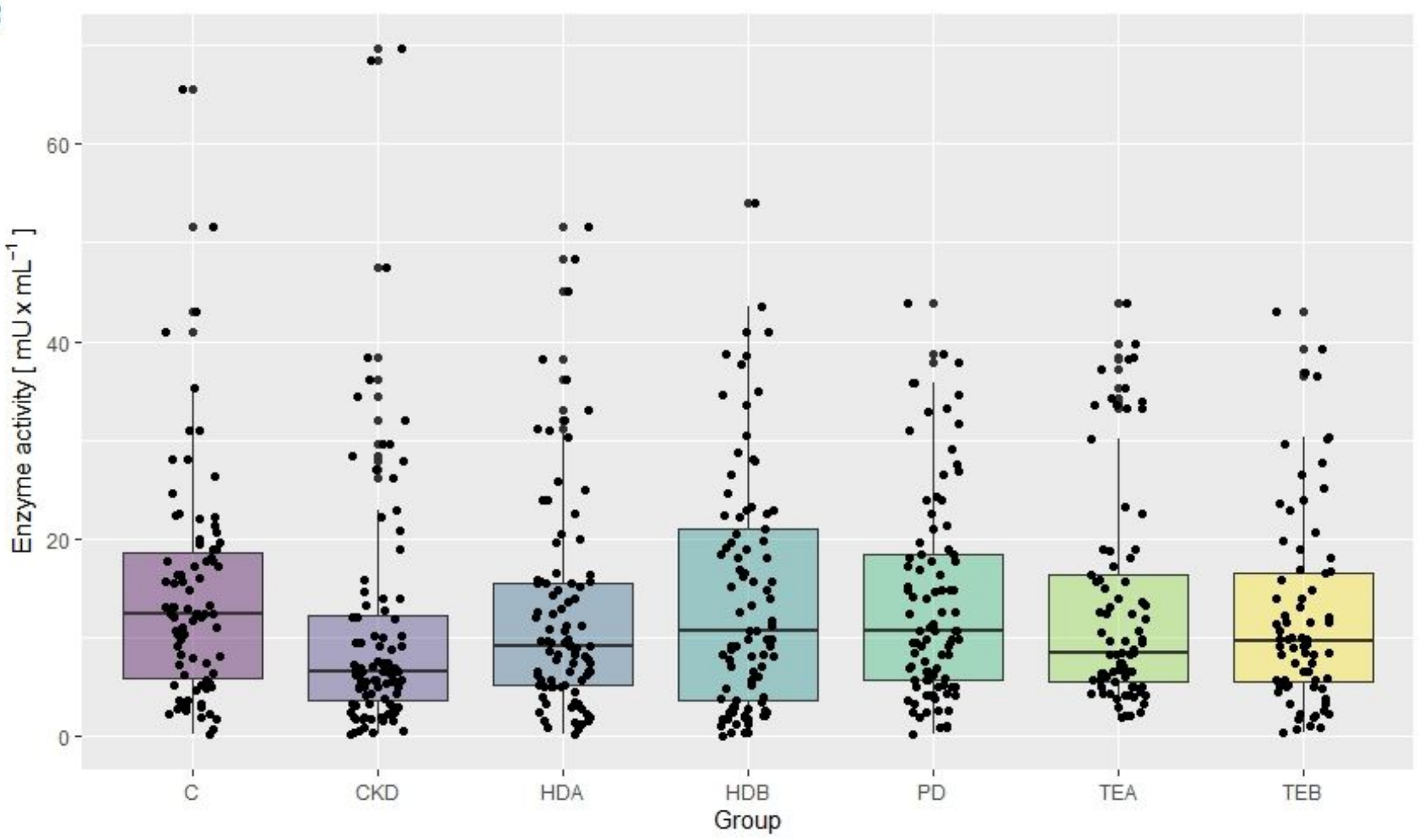

Figure 1

Activity of xanthine oxidoreductase isoforms in A) platelet-rich plasma, B) or platelet-poor plasma. C control group; CKD - treated conservatively; HD B - before hemodialysis; HD A - after hemodialysis; PD - peritoneal dialysis; TE B - before kidney transplantation: TE A - after kidney transplantation. 
A

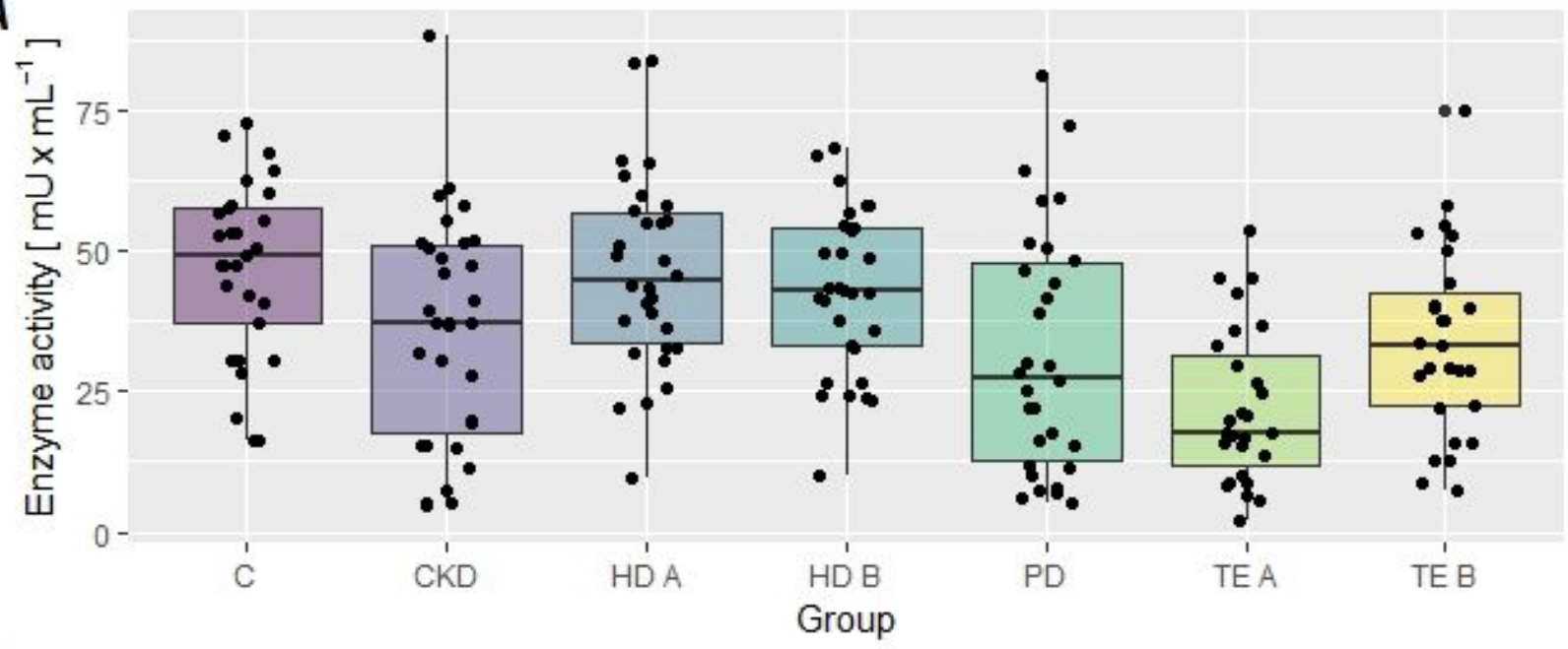

B
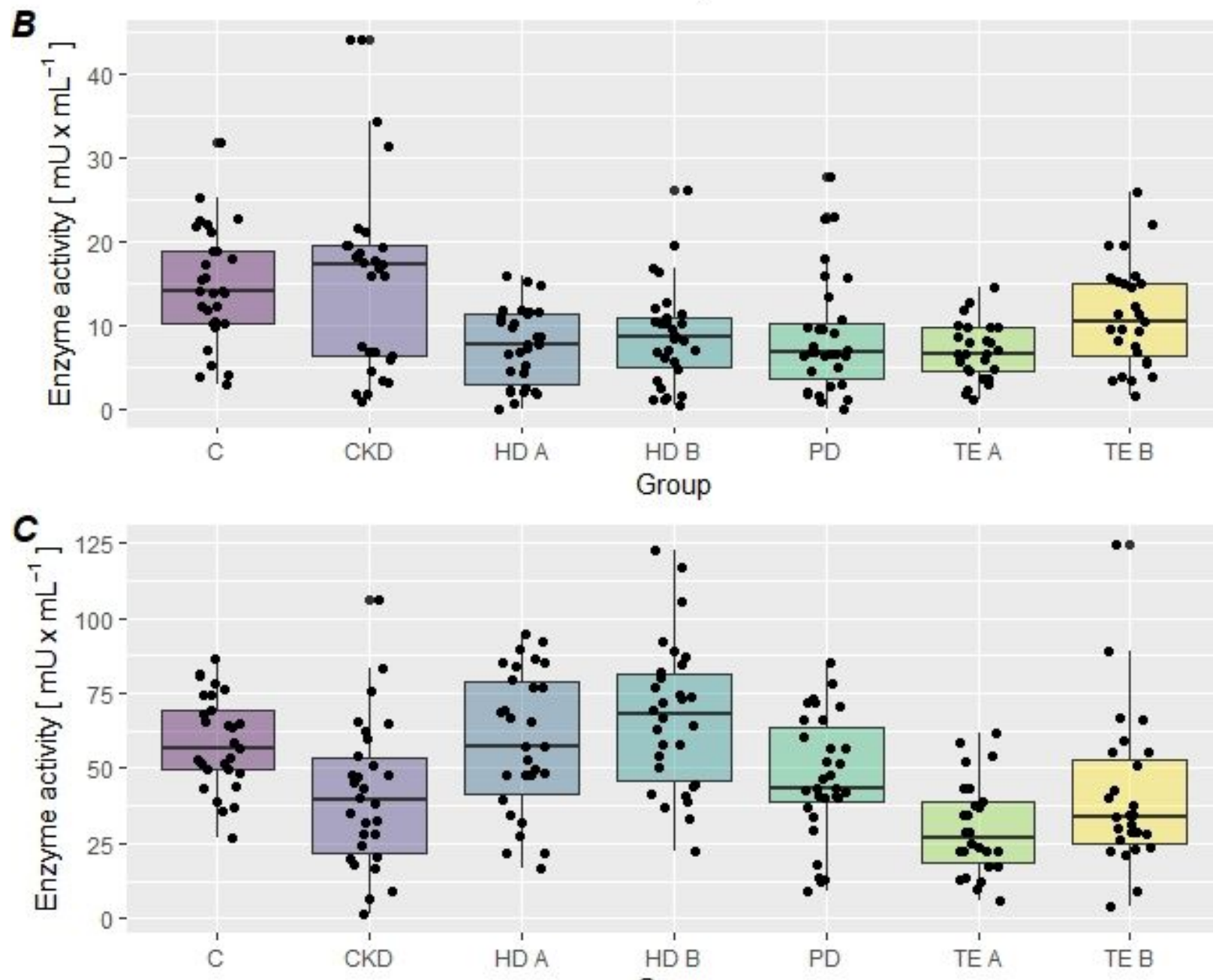

HD B
Group
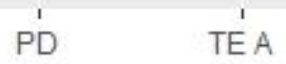

TE B

\section{Figure 2}

Activity of xanthine oxidoreductase isoforms in platelet-poor plasma. A) XD activity, B) XDO activity, and C) XO activity. C - control group CKD - treated conservatively; HD B - before hemodialysis; HD A - after hemodialysis; PD - peritoneal dialysis; TE B - before kidney transplantation: TE A - after kidney transplantation. 

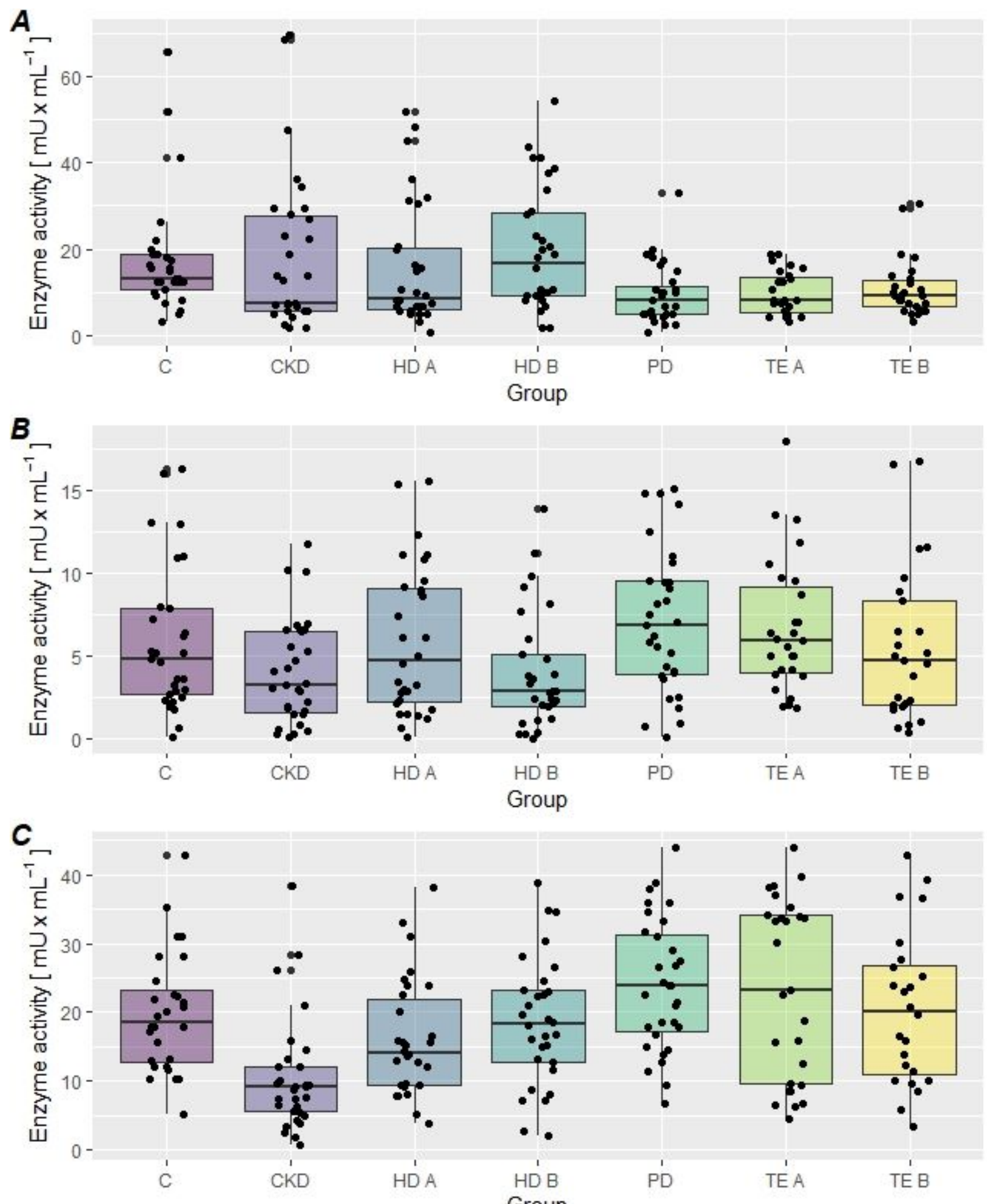

GD B

TE'A

TE B

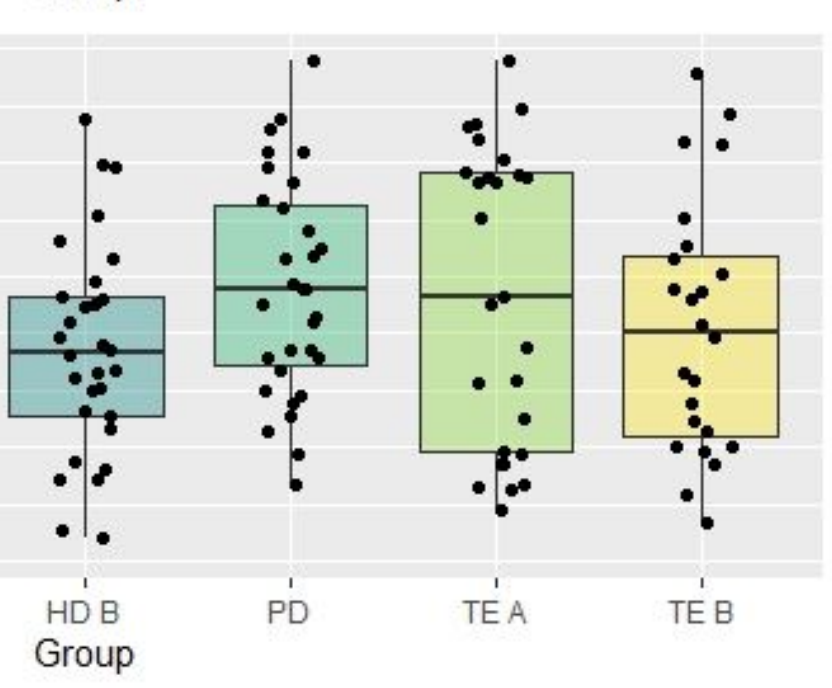

Figure 3

Activity of the xanthine oxidoreductase isoforms in platelet-rich plasma. A) XD activity, B) XDO activity, and C) XO activity. $C$ - control group CKD - treated conservatively; HD B - before hemodialysis; HD A after hemodialysis; PD - peritoneal dialysis; TE B - before kidney transplantation: TE A - after kidney transplantation. 